\title{
Centrality properties of directed module members in social networks
}

\author{
Péter Pollner ${ }^{\mathrm{a}}$ \\ Gergely Palla ${ }^{a}$ \\ Dániel Ábel ${ }^{b}$ \\ András Vicsek ${ }^{\mathrm{c}}$ \\ Illés J. Farkas ${ }^{a}$ \\ Imre Derényi ${ }^{b}$ \\ Tamás Vicsek ${ }^{\mathrm{a}, \mathrm{b}, *}$ \\ ${ }^{a}$ Statistical and Biological Physics Research Group of Hungarian Academy of \\ Sciences \\ 1117 Budapest, Pázmány P. stny. 1/A \\ ${ }^{\mathrm{b}}$ Department of Biological Physics, Eötvös University \\ 1117 Budapest, Pázmány P. stny. 1/A \\ ${ }^{\mathrm{c}}$ Outline Foundation \\ 1023 Budapest, Frankel Leó u. 21-23. II/7
}

\begin{abstract}
Several recent studies of complex networks have suggested algorithms for locating network communities, also called modules or clusters, which are mostly defined as groups of nodes with dense internal connections. Along with the rapid development of these clustering techniques, the ability of revealing overlaps between communities has become very important as well. An efficient search technique for locating overlapping modules is provided by the Clique Percolation Method (CPM) and its extension to directed graphs, the CPMd algorithm. Here we investigate the centrality properties of directed module members in social networks obtained from e-mail exchanges and from sociometric questionnaires. Our results indicate that nodes in the overlaps between modules play a central role in the studied systems. Furthermore, the two different types of networks show interesting differences in the relation between the centrality measures and the role of the nodes in the directed modules.
\end{abstract}

Key words: network modules, overlapping modules, directed networks, clustering, community finding

PACS: 89.75.Hc, 89.75.Fb, 89.65.Ef, 89.75.Da 


\section{Introduction}

A widespread approach to the analysis of complex social and economic phenomena is to assemble the participating individuals or objects and their interactions into a network (nodes and links) and to infer functional characteristics of the entire system from this static web of connections [1,2,3,4,5]. Over the past few years, several broadly studied large-scale properties of real-world webs have been uncovered, e.g. the broad (scale-free) distribution of node degree [6], overrepresented small subgraphs [7,8] and various signatures of hierarchical/modular organisation [9]. Also, many useful measures have been defined to quantify the importance of the individual nodes in the networks. If a vertex lies on many shortest paths running between other vertices, it plays a central role in information flows [10] and is responsible for the vulnerability of the system [11]. In a social network the actors capable of reaching the others with the lowest number of steps (being the closest to others on average) have the greatest influence [12,13].

In recent years there has been a quickly growing interest in the study of the intermediate-scale network structures as well. These units, made up of vertices more densely connected to each other than to the rest of the network, are often referred to as communities, modules, clusters or cohesive groups [14,15, 16, 17]. In the various types of networks' these groups can represent, communities of people [14,18], functional units in molecular biology [19,20], a set of tightly coupled stocks or industrial sectors in economy [21] etc. Search techniques allowing overlaps between such modules are becoming very important [22,23,24,25,26,27,28,29,30]. Indeed, communities in real-world graphs are often inherently overlapping: each person in a social web belongs usually to several groups (family, colleagues, friends, etc.) and proteins in a protein interaction network may participate in multiple complexes. Modules, and also some small subgraphs, are appropriate for "coarse-graining" complex networks: each module/subgraph can be represented as a node and two such nodes can be linked, if the corresponding modules/subgraphs are connected (or overlap) [17,31,32].

In this paper we study the centrality properties of module members (nodes) in four social networks representing either e-mail connections between people or social links obtained from questionnaires. Both types of data sets can be represented by directed networks, therefore, the modules are located using a directed community finding method, the Directed Clique Percolation Method (CPMd) [33]. Our focus is on the correlations between general single-node properties (e.g. the closeness) and the role/importance of the members in the

\footnotetext{
* Corresponding author

Email address: vicsek@angel.elte.hu (Tamás Vicsek).

URL: http://www.cfinder.org.
} 

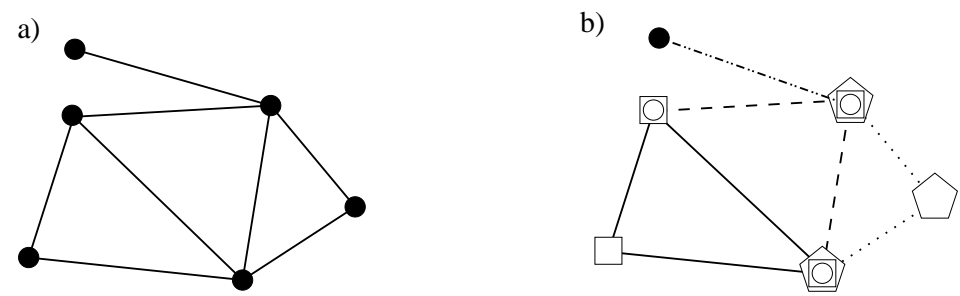

Fig. 1. Visualisation of the CPM method. A community is explored by walking over neighbouring building blocks. The blocks are triangles in this example, that are $k$-cliques with size $k=3$. At figure a) the graph is depicted without marking of blocks. At figure b) the same graph is plotted with marking the nodes with different symbols for each different block. This graph contains three blocks, and one node, which is not a member of any blocks. The nodes of each blocks are marked by circles, rectangles and pentagons respectively. The unclassifiable node is marked by a filled ellipse. The block built from rectangles and the block of circles are neighbours since they differ only in one node. The block of pentagons and circles are neighbours as well. This graph contains only one community, since all blocks are reachable from each other by walking over neighbours. In this case the walks take at most two steps e.g. from block of rectangles to block of circles and than to block of pentagons.

communities, (e.g. the number of communities they participate in).

\section{Directed network modules}

\subsection{Directed clique percolation method (CPMd)}

The CPMd is a natural extension of the Clique Percolation Method (CPM) [17,34]. In the CPM a community is built up from adjacent blocks of the same size $k$. These blocks correspond to $k$-cliques, that are subgraphs with the highest possible density: each pair selected from the $k$ nodes of the subgraph is connected. Two blocks are considered adjacent if they overlap with each other as strongly as possible, i.e., they share $k-1$ nodes. A community is a set of blocks that can be reached from one to the other through a sequence of adjacent blocks 34. Note, that any block belongs always to exactly one community, however, there can be nodes belonging to several communities. (E.g. if blocks overlap only in a single node.) The CPM community searching method is visualised in Fig.1: the communities found by CPM contain only nodes that are densely connected. Nodes with only a few connections or nodes that do not participate in a densely connected subgraph are not classified into any community.

The CPM method is robust against of removal or insertion of a single link. Due to the local nature of this approach, such perturbations can alter only the communities containing at least one of the end points of the link. (In contrast, 
a)

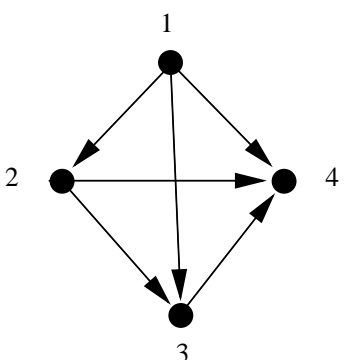

b)

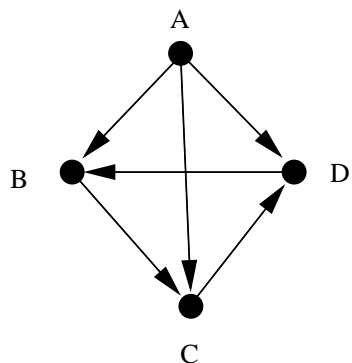

Fig. 2. Two example for directed graphs, that are $k$-cliques of size $k=4$ if neglecting the directionality of the links. However, the graph a) forms a directed $k$-clique, but the graph b) does not. The numbers near the nodes of graph a) show the ordering among the nodes for defining the overall directionality of the clique. The graph $b$ ) cannot be ordered due to the loop formed by the links among nodes $B, C$ and $D$.

for global methods like modularity optimisation the removal or insertion of a single link can result in the change of the overall community structure.)

Finally, we note that the CPM will find the same communities in a given subgraph irrespective to the fact whether the subgraph is linked to a larger network or not. Therefore, a heterogeneous network can be analysed by first dividing it into homogeneous parts, and applying the method to these subnetworks separately. Homogeneous parts of a network can be determined e.g. from external informations like geographical locations of the nodes etc.

The undirected approach detailed above can be made inherently directed by replacing the (undirected) building blocks with directed $k$-cliques. In this Clique Percolation $M$ ethod with directed cliques (CPMd) the building blocks of a community are defined as complete subgraphs of size $k$ in which an ordering can be made such that between any pair of nodes there is a directed link pointing from the node with the higher order towards the lower one [33]. See Fig.2. In the absence of bidirectional links (also called double links) the above condition is equivalent to

- the absence of directed loops (closed directed paths) in the directed $k$ cliques.

- the directionality of all links within the $k$-clique, where any directed link points from a node with a higher order to a lower one.

- the topology, where the number of the nearest out-neighbours (neighbours reached along an out-link) is different for each node in the $k$-clique.

In the presence of bidirectional links a complete subgraph of size $k$ is qualified as a directed $k$-clique if it is possible to rectify the bidirectional links in such a way that the remaining unidirectional links fulfil the above criterion. Note, that without rectification a $k$-clique with a double link cannot satisfy the definition of the directed $k$-clique. One can easily see on Fig 3 , that above conditions cannot fulfilled e.g. there is a loop among the nodes. 
a)

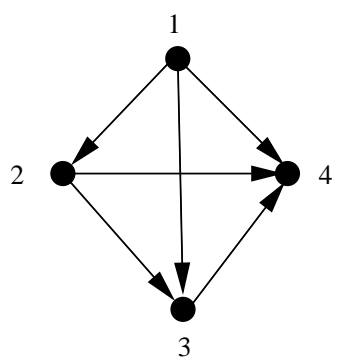

b)

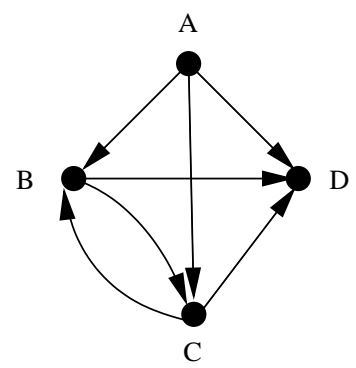

Fig. 3. Two example for directed graphs, that are $k$-cliques of size $k=4$ if neglecting the directionality of the links. The graph a) qualifies as a directed $k$-clique, but the graph b) without rectification does not. The double link between the nodes $B$ and $C$ forms a loop.

The overall directionality of such an object naturally follows the ordering of the nodes: the node with highest order is the one which has only outneighbours, and can be viewed as the "source" or "top"-node of the $k$-clique, whereas the node with lowest order has only incoming links from the others, and corresponds to a "drain" or "bottom" node.

The communities are built up similarly to the undirected case: two directed building blocks ( $k$-cliques) are considered to be neighbours if they share $k-1$ nodes. A directed $k$-clique module (the CPMd modules) arises as the union of directed $k$-cliques that can be reached from each other through a series of neighbouring $k$-cliques.

There are many other possibilities to define a directed $k$-clique. We have used one particular choice 33], which is more restrictive as other natural possibilities, and which is suitable for any $k$-clique without need of further considerations. Previous results showed that even this restrictive definition can result in similar community structures as the undirected method 33$]$.

\subsection{Quantifying the ranking of directed module members}

A natural and simple approach to rank nodes in a module is to compare the number of their incoming and outgoing links. For example, a node having only out-neighbours can be viewed as a "source", whereas a node with only incoming links as a "drain". Most nodes, however, fall between these two extremes. To quantify this property, we use the relative out-degree of node $i$ in module $\alpha$ as

$$
D_{i, \text { out }}^{\alpha}=\frac{d_{i, \text { out }}^{\alpha}}{d_{i, \text { in }}^{\alpha}+d_{i, \text { out }}^{\alpha}}
$$


where $d_{i \text {,in }}^{\alpha}$ and $d_{i \text {,out }}^{\alpha}$ denote the numbers of in-neighbours and out-neighbours amongst the other nodes in the module, respectively [33].

Obviously, the values of $D_{i \text {,out }}^{\alpha}$ fall into the range between 0 (for drain nodes) and 1 (for source nodes). The analogous quantity for in-degrees is obtained as $1-D_{i \text {,out }}^{\alpha}$. Since modules may overlap, nodes may have as many relative out(in)-degree as many modules they participate in.

\section{Centrality measures of individual nodes}

In the following we shall use the betweenness centrality [35] and the closeness centrality [13] to characterise the role and position of the nodes in the studied networks. We apply these measures to directed networks, where moving along an edge is allowed only into the direction of the edge. This restriction effects the possible shortest paths and the distance of nodes as well.

The betweenness centrality, $b_{i}$, of node $i$ measures the importance of $i$ in the communication between any node pair in the network [12]. We use the definition

$$
b_{i}=\sum_{l \neq i \neq j} \sigma_{l j}(i)
$$

where $\sigma_{l j}(i)$ is the portion of number of all shortest paths between node $l$ and node $j$ that go through $i$. The closeness centrality, $c_{i}$, of node $i$ is defined as

$$
c_{i}=\frac{1}{\sum_{j} d(i, j)},
$$

where $d(i, j)$ is the distance between $i$ and $j$. The closeness centrality of a node is large if it can be reached within a small number of steps on average. Note that in directed networks the distance (the number of steps along a shortest path) may depend on the direction of the path. Therefore, we shall differentiate between in- and out-closeness $\left(c_{i, \text { in }}\right.$ and $\left.c_{i \text {,out }}\right)$ depending on whether $i$ is the end point or the starting point of the path connecting $i$ and $j$.

\section{Results in social networks}

In this section we examine the centrality properties of nodes in directed social networks with respect to their membership number $m$ (the number of modules they participate in) and with respect to their relative out-degrees. The 
networks under study represent the directed e-mail connections between the students at the University of Kiel [36], (containing 57, 158 nodes and 103, 701 links), the e-mails between the employees of the Enron concern [37], (consisting of 151 nodes and 1364 links), and two networks obtained from sociometric questionnaires in a smaller and a medium-sized company. In the latter cases a directed link was set between two employees if one has named the other in any question 1 , resulting in 1166 links between 115 employees for the small company and 4349 links between 597 nodes for the other company.

When extracting the directed modules using the CPMd, we adjusted the parameter $k$ ( $k$-clique size) using a criterion to find a modular structure as highly structured as possible. This criterion is based on the observation, that for many real networks there is a well defined parameter range, where no giant community (having size in order of magnitude as the size of the whole network) can be found, instead many smaller communities occur. However, if the parameters are changed (in our case the value of $k$ is decreased), the rich community structure disappears and one giant community dominates the network. In the optimal setting the parameters are adjusted just below the critical point of this percolation transition (for details see [17]).

For data sets studied in this paper the percolation threshold can be determined by observing the change in the size, $s$, of the largest community as the control parameter (the clique size, $k$ ) increases. Since there is no weight defined on the links in our networks, we have only one control parameter. Below the percolation threshold (at small $k$ values) a giant community exists in the network. If $k$ increases, near the critical point the size of the largest community drops quickly. At large $k$ values the size of the largest community decreases at a moderate rate.

In Fig, 4 we show the behaviour of the size of the largest community. Since the size of the largest community, $s$, is different for each network, we have plotted a rescaled community size, $s^{*}$, equal to $s$ divided by the size of the largest community at $k=3$. (This way, each curve starts with $s^{*}=1$ at the smallest possible $k$, which is $k=3$.) We found, that the optimal $k$ value for the Enron data set and for the questionnaire data set of the smaller company is $k=6$. For the larger company we found the optimal value $k=5$, whereas for the email data set of the University of Kiel it turned out to be $k=4$.

$\overline{1}$ In a questionnaire there are several questions concerning different type of social relations, and answers are typically names of colleagues who are thought to satisfy the posed situation. 


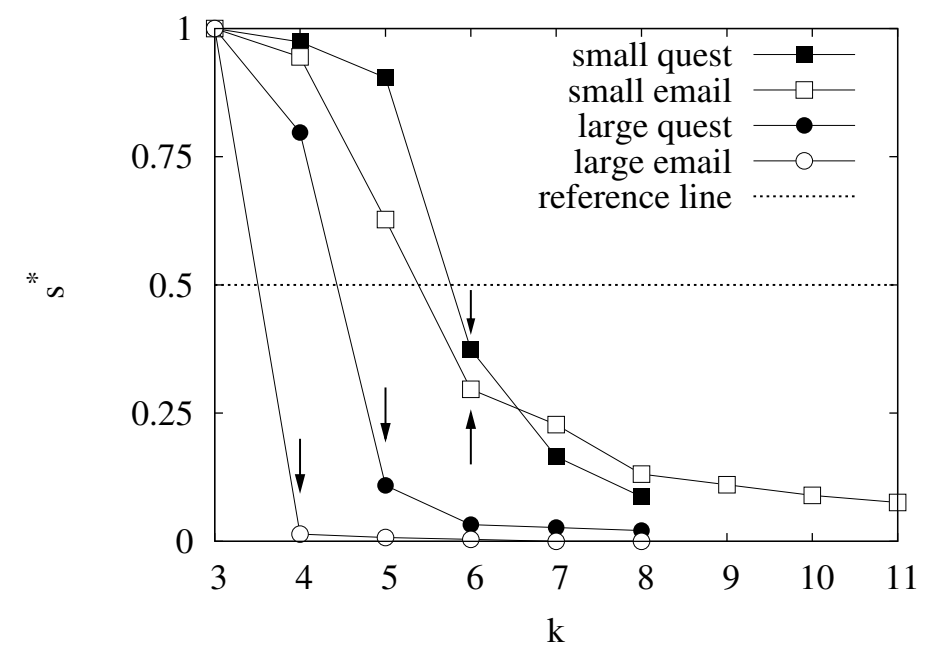

Fig. 4. The rescaled size, $s^{*}$, of the largest community decreases with increasing $k$. Near the percolation threshold $s^{*}$ decreases rapidly, and the optimal $k$ value (marked by a small arrow) is chosen to be the smallest $k$ where no giant community can be found in the network.

\subsection{Extracting networks from email and questionnaire data}

There is a growing interest of measuring and analysing social relation data within organisations. Relations between people can be of various types: they can exchange informations, meet each other by lunch, etc.

Nowadays information is mainly exchanged by using some electronic form: either by phone calls 18 or by emails. Email is the electronic form of letters: there is always a sender, whom the email is sent from, and there is a receiver, whom the email is sent to. If one person writes an email to another one, a social relation emerges between two actors. These contacts can be represented by a network in which the nodes correspond to the users and the links to the emails. These connections are directed, and the natural choice for the direction is to follow the path of the email, pointing from the sender to the receiver.

The same message content can be spread among several recipients by listing more than one recipient in the header. We consider this type of contacts as several individual relations originating from the sender to each of the receivers. Here we do not analyse the correlations emerging from emails with multiple recipients.

Other, less formal relations (e.g. someone can trust in the opinion of others) are hard to measure directly and are practically impossible to extract from automatically recorded resources. Thus, one has to apply indirect measuring methods. The most often used solution is provided by questionnaires. In questionnaires the investigated set of people (e.g.employees of a company) are 

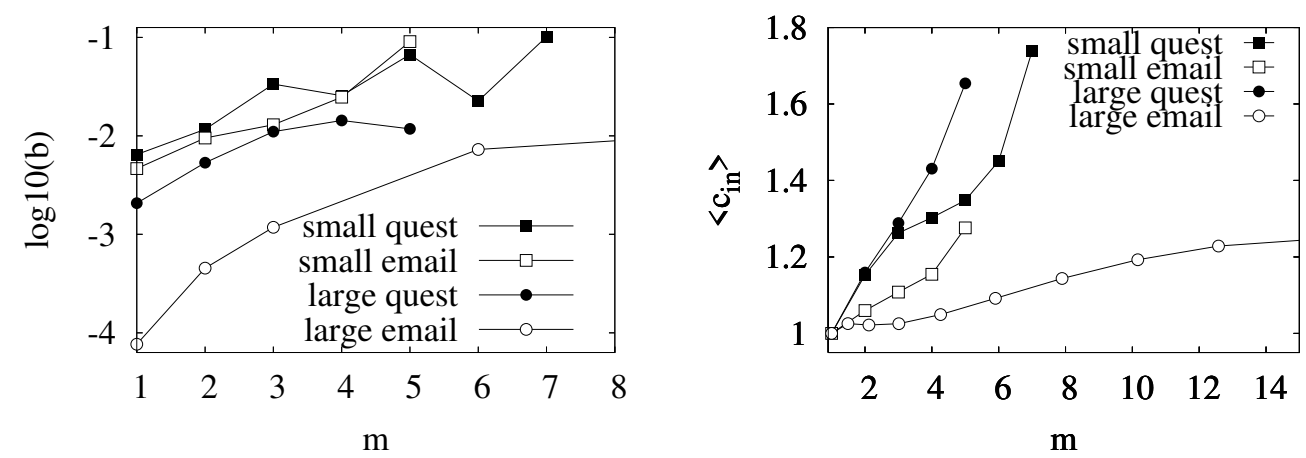

Fig. 5. Left: betweenness as a function of the membership number. Due to the logarithmic scale nodes with zero betweenness are dropped. The smooth lines show the tendency of the data. Right: smoothed average in-closeness against the membership number. (For clarity the lines are scaled to start at 1). Both centrality measures show increasing tendency in function of $m$.

asked the same questions relating to their social contacts. These questions are formulated along the rules of standard sociometry: they must take into account, that the answerers are human beings with restricted time and memory capacity as compared to computers. Humans can have different interests and can cheat in their answers. Well organised questionnaires can help to minimise false results due to such effects.

We used results of two surveys, where the employees received a set of questions concerning their social contacts. The answers to the questions contained a list of names, where the names had to be picked out from a prepared list of colleagues. The network representation was built up from the questionnaire results by representing the employees as nodes. Two nodes are connected, if there exists an answer in any of the questionnaires, that justifies the existence of a social relation between the corresponding two employees. If an employee marked another employee in any of the answers then a directed link points from the answerer to the colleague named in the answer.

\subsection{Nodes in community overlaps are central}

After extracting the directed modules using the CPMd, we first examined the centrality properties of the nodes in the overlaps between the modules (the nodes having a membership larger than one).

In Fig. 5 we show $\left\langle b_{i}\right\rangle$ and $\left\langle c_{i, \text { in }}\right\rangle$ as a function of the membership number $m$. We can observe a clear tendency: nodes participating in more communities are more central. They can control more effectively the flow of information, since they lie typically on more shortest paths. Also, they can reach other nodes faster, since they need less steps on average to others. 


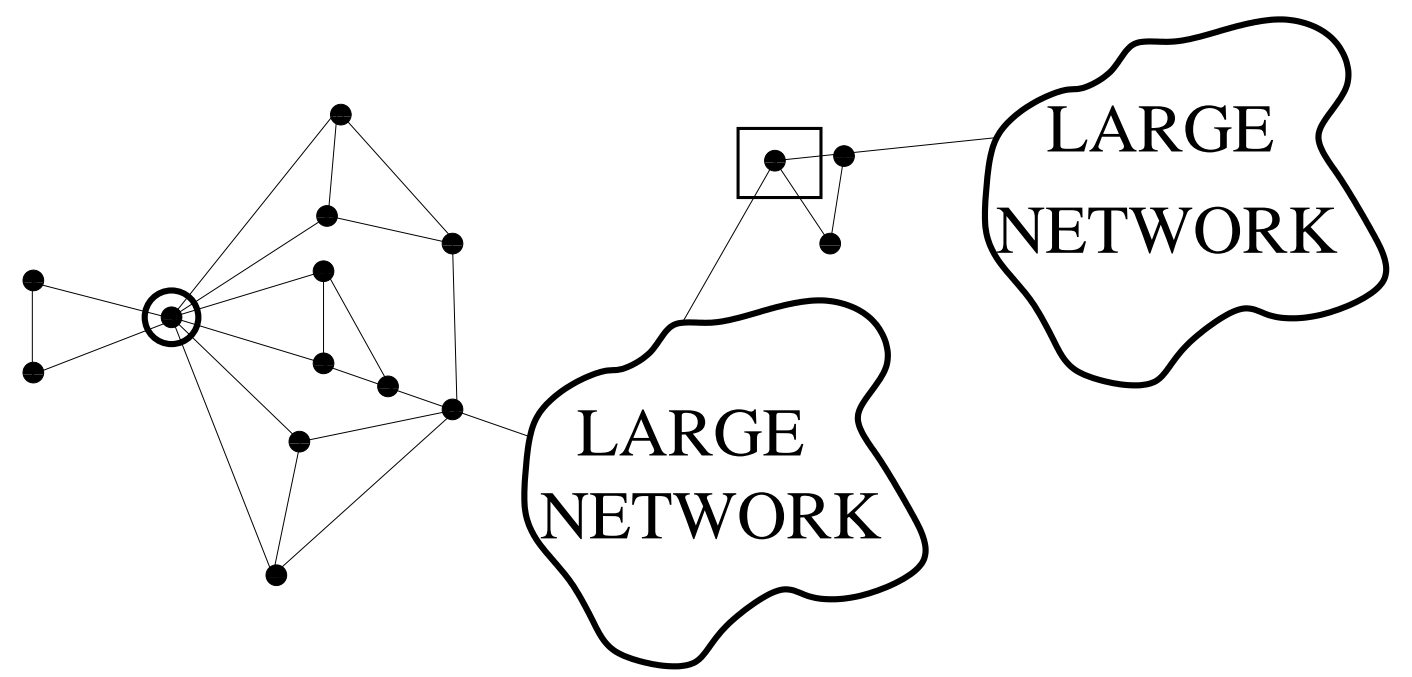

Fig. 6. Counter-example for the correlation between the membership and centrality measures. The node marked by a surrounding circle has a large membership number: at $k=3$ its membership number is $m=5$ and the betweenness and closeness value of this node is small, since this node is at the periphery of the network. However, the node marked by a frame has a membership number $m=1$ and its betweenness is large, since it acts as a bridge.

Note, that the observed positive correlation between the centrality measures and the membership number is not trivial. It is easy to construct counterexamples, where nodes with high membership number are at the periphery of the network. We show a sketch of such a network in Fig. 6. Similar counterexamples can be generated for any $k$.

\subsection{Centrality as a function of the relative out-degree}

Next we analysed the dependence of the two chosen centrality measures on the relative out-degree of the module members. According to Fig. 7 both the betweenness centrality and the in-closeness show a decreasing tendency with increasing $D_{i \text {,out }}^{\alpha}$ in the networks obtained from questionnaires (black symbols). In other words: people named by fewer colleagues than vice versa are less central in the network. These people can be reached by others in more steps on average (they have smaller in-closeness values), and they have less control on the information flow (their betweenness centrality is lower). Interestingly this tendency cannot be observed in case of the e-mail networks (white symbols in Fig. 7), where the centrality measures are more or less independent of the relative out-degree (or show a slight increasing tendency). For all four networks $\left\langle c_{i, \text { in }}\right\rangle$ suddenly drops to zero at $D_{i, \text { out }}^{\alpha}=1$. This is due to the fact that nodes having only out-neighbours have a zero in-closeness by definition. 

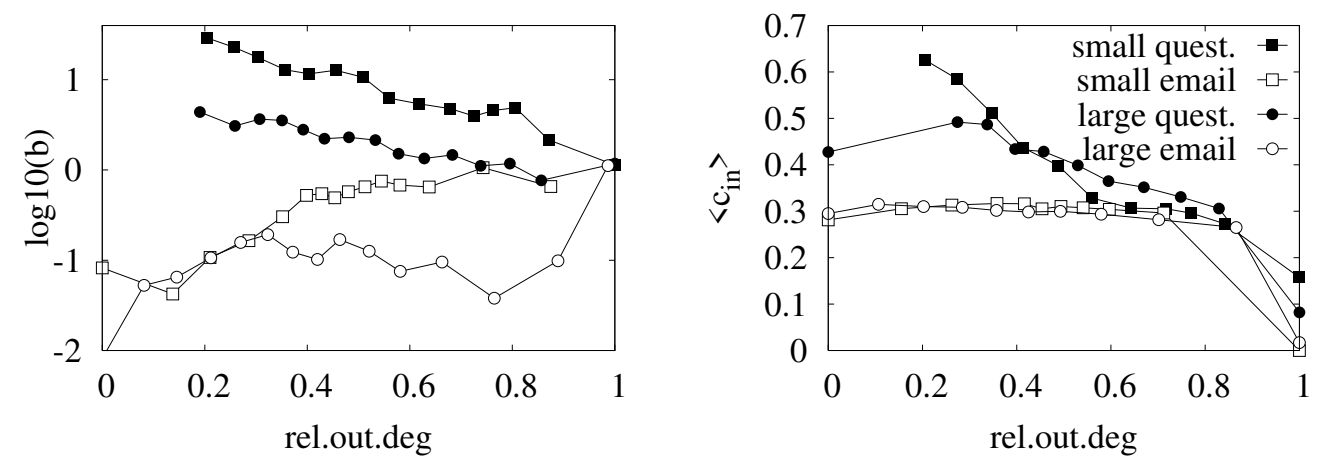

Fig. 7. Left: relative out-degree and betweenness. The smoothed curves are shifted to 0 at the maximal values. Again, e-mail networks do not show decreasing tendency as questionnaire networks do. Right: relative out-degree and averaged in-closeness. The $\left\langle c_{i, \text { in }}\right\rangle$ is decreasing with $D_{i \text {,out }}^{\alpha}$ for the questionnaire networks, whereas it is more or less constant for the e-mail networks. For better visibility the smoothed in-closeness values are scaled to take similar values before the cutoff.

This difference between the two types of networks is likely to originate in the different methods used to collect the data. E-mail networks are generated automatically, no interaction with the observed persons is necessary. In contrast, a questionnaire can not be filled without some interaction. Interaction needs time from the respondent and from the interviewer as well. This sets some restrictions to the number of emerging links: the number of questions and the number of possible answers can not be as large as the number of e-mails between actors that can be exchanged during several months. Furthermore, emails are collected in a wide time span (during which fluctuations are averaged out), whereas a questionnaire captures an instantaneous state.

\subsection{Average membership as a function of the relative out-degree}

Another interesting difference between the studied email and questionnaire networks can be observed in the relation between the average membership of a node (the average number of communities the node participates in) and the relative out-degree (11). In questionnaire networks the average membership takes larger values at nodes whose relative out-degree is small. In contrast, we find the membership maximum at large relative out-degrees for nodes in email networks. The corresponding curves are shown in Fig, 8 .

A plausible explanation of this observation can be that in email networks the directionality of the links follows the direction of the information flow: there is always some content in the text body of the email. Those persons, that participate in several groups (communities) will probably more often forward information between communities: if some knowledge is available only in one community, than the actors in the community overlaps will provide this 


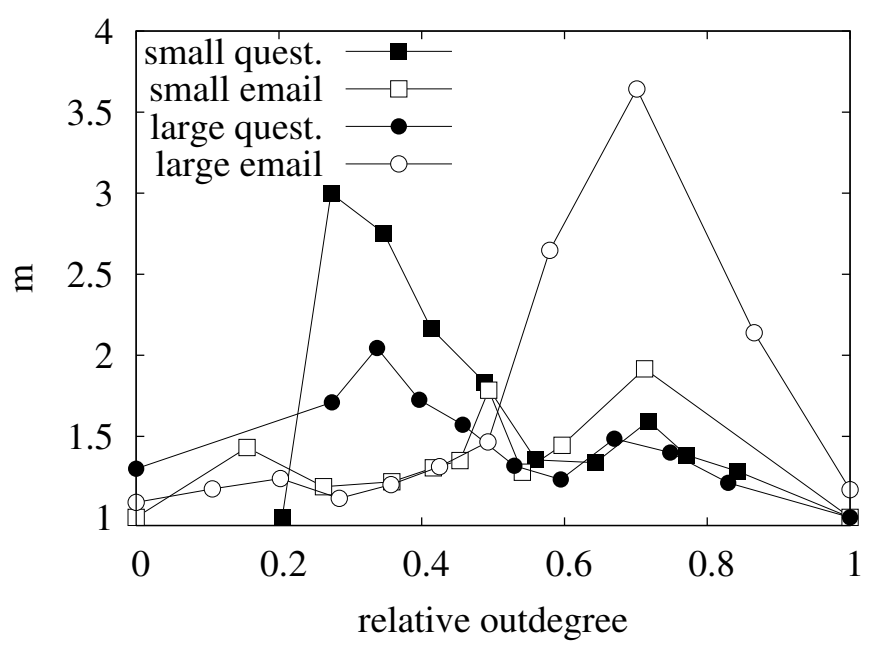

Fig. 8. Relative out-degree and membership: the averaged membership takes its maximum at lower relative out-degree in questionnaire networks and it is maximal at high relative out-degree for email networks.

information for the other communities they participate in.

In questionnaire networks the direction is opposite: if one has named somebody he will probably ask him to receive some information. Hence the direction of the links are the opposite as of the information flow: the most often named persons are most trusted for helping or providing helpful information. If a person is named by many others, than its node will have large relative indegree (small relative out-degree). Probably those persons can help others who can easily build new social contacts, and therefore they probably participate in several different communities.

\section{Summary}

We have examined four directed social networks constructed from e-mail exchanges and from questionnaire data. We have extracted the overlapping directed module structure of these nets using the CPMd algorithm, and studied the behaviour of the betweenness centrality and closeness for module members. Our results indicate that nodes with high memberships (participating in numerous modules in parallel) play a central role in these networks. Furthermore, our investigations revealed interesting differences between the two types of networks concerning the dependence of the centrality measures on the relative out-degree of module members (the ratio of out-degree versus number of all nearest neighbours within the modules) . 


\section{Acknowledgements}

The authors thank the partial support of the Hungarian National Science Fund (OTKA T034995, K068669, PD048422, K060456) and the National Research and Technological Office (NKTH, CellCom RET, Textrend). Questionnaire data were generously made available by Outline Foundation.

\section{References}

[1] Barabási, A. L. and Albert, R., Rev. Mod. Phys. 74, 47 (2002).

[2] Dorogovtsev, S. N. and Mendes, J. F. F., Evolution of Networks: From Biological Nets to the Internet and $W W W$ (Oxford University Press, 2003).

[3] Boccaletti, S. et al, Physics Reports 424, 175 (2006).

[4] Newman, M. E. J., SIAM Review 45, 167 (2003).

[5] Watts, D. J., Ann. Rev. Soc. 30243 (2004).

[6] Barabási, A.L. and Albert, R., Science 286, 509 (1999).

[7] Milo, R. et al, Science 298824 (2002).

[8] Alon, U., Nat. Rev. Gen. 8, 450 (2007).

[9] Ravasz, E. et al, Science 297, 1551 (2002)

[10] Brandes U., Journal of Mathematical Sociology 25163 (2001).

[11] Holme P. et al, Phys. Rev. E 65, 056109 (2002).

[12] Newman M. E. J., Phys. Rev. E 64, 016132 (2001).

[13] Sabidussi G., Psychometrika 31, 581 (1966).

[14] Scott J., Social Network Analysis: A Handbook, 2nd edn (Sage Publications, London 2000).

[15] Shiffrin R.M. and Börner K., Proc. Natl. Acad. Sci. USA 101, 5183 (2004).

[16] Girvan M. and Newman M. E. J., Proc. Natl. Acad. Sci. USA 99, 7821 (2002).

[17] Palla G., Derényi I., Farkas I. J. and Vicsek T., Nature 435, 814 (2005).

[18] Palla G., Barabási A. L. and Vicsek T., Nature 446, 664 (2007).

[19] Spirin, V. and Mirny, L. A., Proc. Natl. Acad. of Sci. U S A 21, 12123 (2003).

[20] Ihmels, J., et al, Nat. Genet. 31, 370 (2002).

[21] Onnela J.P. et al, Phys. Rev. E 68, 056110 (2003). 
[22] Wilkinson, D.M., Huberman, B.A. Proc. Natl. Acad. Sci. USA 101, 5241(2004)

[23] Baumes, J., Goldberg, M., Magdon-Ismail, M. Lect. Notes in Computer Science 3495, 27(2005)

[24] Guldener, U., Munsterkotter, M., Kastenmuller, G., Strack, N., van Helden, J. Nucl. Ac. Res. 33, D364(2005)

[25] Vicsek, T. Physica A-Statistical Mechanics and its Applications 378, 20(2007)

[26] Gfeler, D., Chappelier, J.C., Rios, P.D.L. Phys. Rev. E 72, 056135 (2005)

[27] Zhang, S., Wang, R.S., Zhang, X.S. Phys. Rev. E 76, 046103 (2007)

[28] Newman, M.E.J., Leicht, E.A. Proc. Natl. Acad. Sci. USA 104, 9564(2007)

[29] Reichardt, J., Bornholdt, S. Phys. Rev. Lett. 93, 218701 (2004)

[30] Nepusz, T., Petróczi, A., Négyessy, L., Bazsó, F. Phys. Rev. E 77, 016107 (2008)

[31] Song C., Havlin S. and Makse H. A., Nature 433, 392 (2005).

[32] Pollner P., Palla G. and Vicsek T., Europhys. Lett. 73, 478 (2006).

[33] Palla G. et al, New Journal of Physics 9, 186 (2007).

[34] Derényi I., Palla G. and Vicsek T., Phys. Rev. Lett. 94, 160202 (2005).

[35] Freeman L. C.: Sociometry 40, 35 (1977).

[36] Ebel H., Mielsch L. I. and Bornholdt S., Phys. Rev. E 66, 035103 (2002).

[37] Shetty J. and Adibi J., Enron email dataset database schema and brief statistical report. Technical report, USC Information Sciences Institute, (2004). 